\title{
Robust Skin Color Segmentation Using a 2D Plane of RGB Color Space
}

\author{
Juneho Yi, Jiyoung Park, Jongsun Kim, and Jongmoo Choi \\ School of Information and Communication Engineering \\ Sungkyunkwan University \\ Suwon 440-746, Korea \\ \{jhyi, jypark, jskim, jmchoi\}@ece.skku.ac.kr
}

\begin{abstract}
This research features a new method for skin color segmentation using a $2 \mathrm{D}$ plane in the RGB color space. The RGB color values of the input color image do not need to be converted into HSI or YIQ color coordinates that have popularly been used for color segmentation. We have observed an important fact that skin colors in the RGB color space are approximately distributed in a linear fashion. Based on this fact, we have applied PCA (Principal Component Analysis) techniques to RGB values of skin colors from a set of training images. We detect skin regions by the lookup of skin color histogram computed based on a $2 \mathrm{D}$ color plane of which two axes correspond to two directions with smallest spread of skin colors.
\end{abstract}

The proposed 2D color plane for color histogram lookup has an advantage over HS or IQ color planes. By using this plane, the problem of color constancy is much relieved. A learned color histogram contains most skin colors detected in the input images and at the same time, the distribution of skin colors in the plane is invariant compared to those in the HS or IQ planes. We have evaluated the performance of the proposed method by comparing with the performance of color histogram lookup methods based on HS or IQ color plane. The experimental results show that the performance of our method is robust to illumination changes.

\section{Introduction}

Face and hand gesture recognition is critical to the realization of natural humancomputer interfaces based on computer vision. In order to recognize a face or gesture, skin regions should be first detected in the input image. An efficient way of doing this is color segmentation and a practical method is to employ a 2D lookup table of skin colors based on a learned color histogram [1,2].

Color segmentation based on a RGB color space is known to be sensitive to illumination changes. In most cases, the RGB color space is converted to HSI [7-10] or YIQ [11] color spaces where the luminance and the chrominance components of a color are separated. Two dimensional subspaces such as the HS plane of HSI color space and the IQ plane of YIQ color space that correspond to the chrominance 
component (I of HIS or Y of YIQ) have usually been used for building a color histogram because the luminance component is very sensitive to illumination changes.

One of the most desirable properties that a 2D color plane for color histogram must satisfy is as follows. The distribution of skin colors in a learned color histogram should not be much variant. At the same time, the learned color histogram should contain most skin colors detected in the input images. If a 2D color plane that is used for the color histogram provides this property, we rarely need to update the histogram. In the case of illumination changes, the distribution of skin colors in the HS or IQ planes is very variant. Figure 1 shows a color histogram of $\mathrm{H}$ (hue) and $\mathrm{S}$ (saturation) values of the same skin region under various lighting conditions due to illumination or hand pose changes. The distributions of $\mathrm{H}$ and $\mathrm{S}$ values of the same skin region are quite different. This makes the use of a color histogram without its update for skin color segmentation very hard [9, 12]. It is quite probable that HS or IQ values of many skin pixels in the input image do not belong to the skin color regions of the learned color histogram and that those pixels are classified as non-skin pixels. In most cases, color histograms based on the HS or IQ plane need to be updated in order to give performance robust to illumination changes.
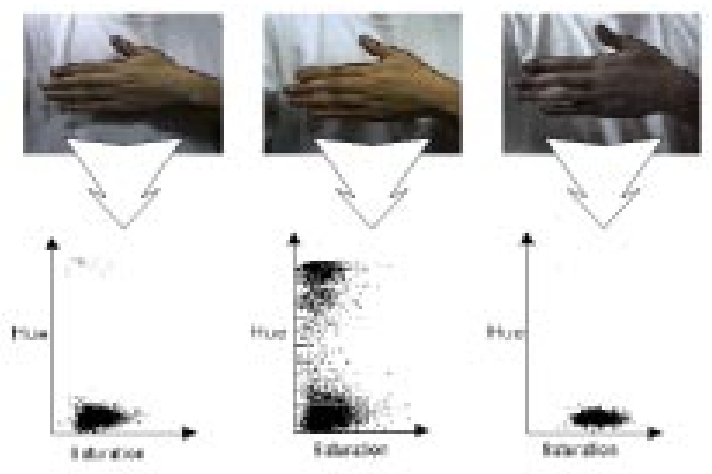

Fig. 1. Histograms of $\mathrm{H}$ and $\mathrm{S}$ values of the same skin region under various lighting conditions due to illumination or hand pose changes

We propose a 2D color plane of RGB color space that performs better than commonly used HS or IQ color planes for color segmentation. The idea is based on the fact that skin colors in the RGB color space are approximately distributed in a linear fashion [3-6]. We have observed ourselves this fact through experiments as can be seen in Fig 5. We have applied PCA techniques to RGB values of skin colors from a set of training images. We detect skin regions by the lookup of skin color histogram computed based on a 2D color plane of which two axes correspond to two directions with smallest spread of skin colors. In the color histogram based on the proposed 2D plane, the distribution of skin colors form a tight cluster in a small region and is relatively invariant in case of illumination changes. Thus, the problem of color constancy is less severe than when HS or IQ planes are used. This alleviates the need to update the color histogram. In addition, at the time of on-line detection, RGB pixel 
values do not need to be converted into another color coordinates such as HSI and YIQ, resulting in saving of computation time and space. We have evaluated the performance of the proposed plane by comparing with the performance of color histogram lookup methods based on HS or IQ color plane using images under varying illumination conditions. The Experimental results show that our method gives a good segmentation performance robust to illumination changes.

This paper is organized as follows. The following section briefly reviews related work. Section 3 presents the proposed method. Experimental results are reported in section 4.

\section{Related Work}

A practical method for skin color segmentation is to employ a lookup table (i. e. 2D color histogram) of skin colors [1,2] where the two axes of the lookup table correspond to the chrominance component of an appropriate 3D color space. Most commonly used color planes for building color histograms are the HS plane of HSI color space and the IQ plane of YIQ color space as displayed in Fig. 2.

Lookup table methods typically process the input image pixel by pixel for segmentation. In off-line, a two-dimensional lookup table of chrominance components is computed from training images by using an appropriate color space. In on-line, a pixel of the input image is simply classified as a skin pixel if it is one of the entries in the lookup table computed off-line.

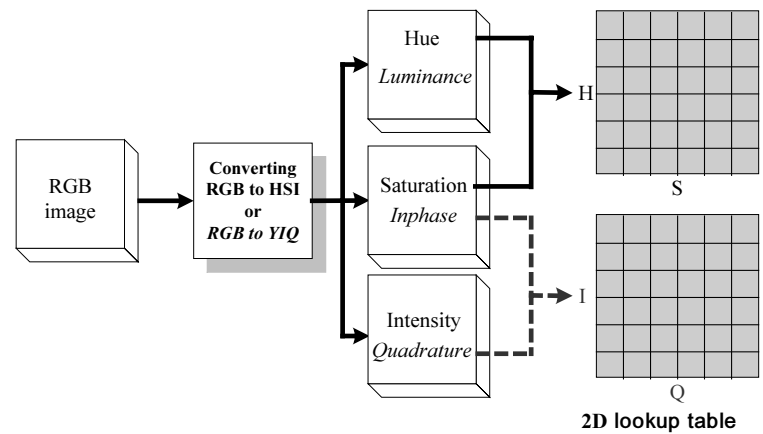

Fig. 2. 2D color planes, HS and IQ, for building a color lookup table (i. e. color histogram)

\section{The Algorithm Overview}

We have implemented a simple algorithm for skin color segmentation to evaluate the performance of the proposed 2D color plane for color histogram. The algorithm 
processes the input image pixel by pixel for segmentation. The algorithm is shown in Fig. 3.

In off-line, we apply PCA techniques to RGB values of skin colors from a set of training images and get a linear projection matrix. We build a $2 \mathrm{D}$ color histogram based on a plane of which two axes correspond to directions of smallest spread of RGB values of skin pixels. The vectors representing these two directions are the column vectors of a linear projection matrix. In on-line, a pixel of the input image is simply projected using the linear projection matrix computed off-line and simply classified as a skin pixel if it is one of the entries in the lookup table.

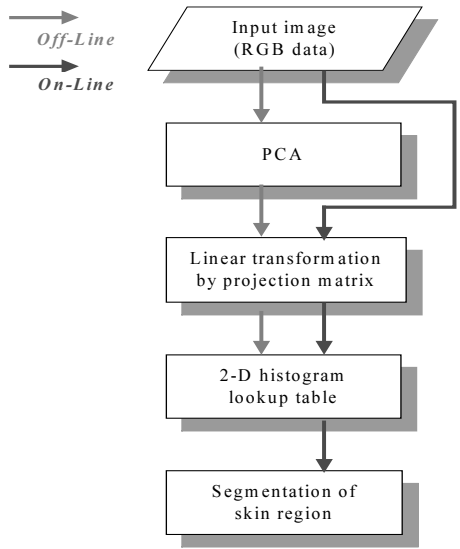

Fig. 3. A simple color histogram lookup algorithm for skin color segmentation to evaluate the performance of the proposed 2D plane for color histogram

\subsection{Principal Component Analysis}

Let $X$ be a set of RGB values of pixels that belong to skin regions collected off-line.

$$
X=\left[\overrightarrow{X_{1}}, \overrightarrow{X_{2}}, \ldots \ldots \ldots, \overrightarrow{X_{T}}\right], T=\text { the number of training images }
$$

The mean vector, $\vec{M}$, is computed as $\vec{M}=\sum_{i=1}^{T} \vec{X}_{i}$ and we get $\Phi$ by subtracting $\vec{M}$ from $\vec{X}_{i}$ 's:

$$
\Phi=\left[\overrightarrow{\Phi_{1}}, \overrightarrow{\Phi_{2}}, \ldots \ldots . ., \overrightarrow{\Phi_{T}}\right]
$$

where $\vec{\Phi}_{i}=\vec{X}_{i}-\vec{M}$. The covariance matrix, $S_{T}$, is computed as: 


$$
S_{T}=\sum_{i=1}^{T} \vec{\Phi}_{i} \vec{\Phi}_{i}^{T}=\Phi \Phi^{T}
$$

The eigenvalues and eigenvectors of $S_{T}$ are obtained using equation (4).

$$
S_{T} \Psi=\Psi \Lambda
$$

where $\Psi=\left[\vec{\psi}_{1}, \vec{\psi}_{2}, \vec{\psi}_{3}\right]$ and $\Lambda=\left[\lambda_{1}, \lambda_{2}, \lambda_{3}\right]\left(\lambda_{1} \geq \lambda_{2} \geq \lambda_{3}\right)$ represent the eigenvectors and eigenvalues, respectively.

Two eigenvectors, $\vec{\psi}_{2}$ and $\vec{\psi}_{3}$, that correspond to smallest eigenvalues, $\lambda_{2}$ and $\lambda_{3}$, represent two directions with smallest spread of $\vec{X}_{i}$ 's (i. e. RGB values of skin pixels). A $2 \mathrm{D}$ lookup table (i. e. color histogram) is built by projecting all $\vec{X}_{i}$ 's onto the $2 \mathrm{D}$ plane defined by the two axes, $\vec{\psi}_{2}$ and $\vec{\psi}_{3}$, as in equation (5). This lookup table is loaded for online detection of skin colors. $W_{p c a}$ is a linear projection matrix of which two column vectors are $\vec{\psi}_{2}$ and $\vec{\psi}_{3}$.

$$
\vec{Y}_{i}=W_{p c a}^{T} \overrightarrow{X_{i}}
$$

\section{Experimental Results}

For the experiment, we have used facial images. Facial images are captured at three meters away in three different lighting directions under varying lighting intensity. Refer to Fig. 4. Images from frontal and side lighting were used for training and images from $45^{\circ}$ lighting for test.

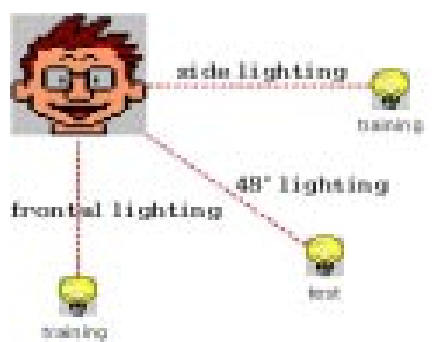

Fig. 4. Facial images are captured in three different lighting directions for the experiment.

Fig. 5 shows a set of example images used in the experiment. Three levels of lighting intensity were used: high, medium and low. Fig. 6 displays the distribution of RGB values of skin pixels in the RGB color space. We can see that the distribution is quite linear and that the first eigenvector from the PCA analysis will be in this direction of the greatest change of RGB values. 


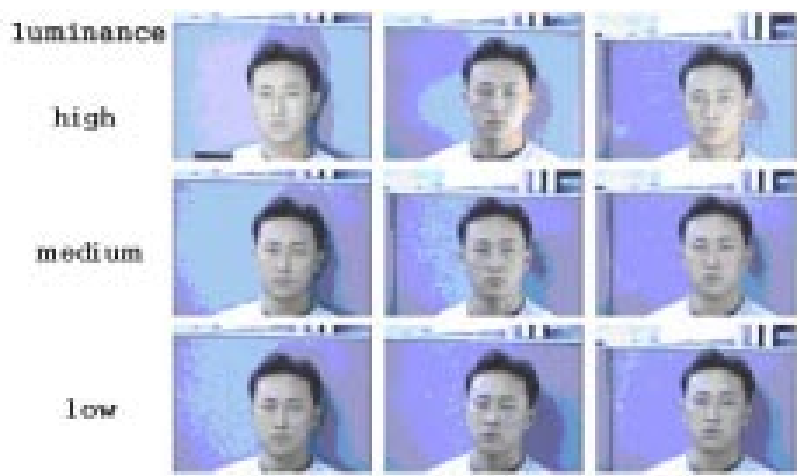

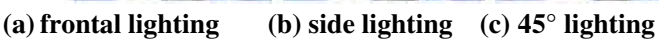

Fig. 5. Examples of images used in the experiment

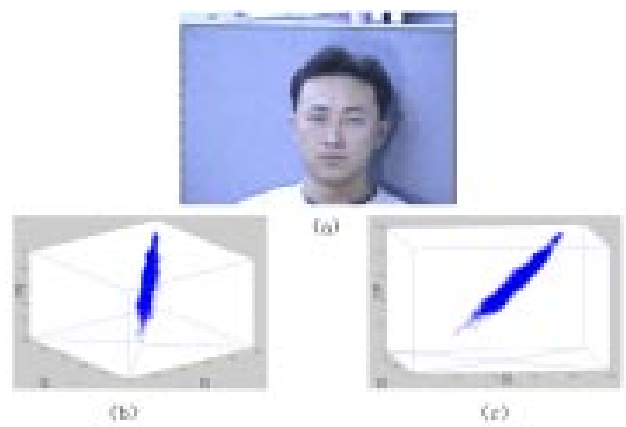

Fig. 6. (a) An example image, (b) (c) the visualization of the distribution of RGB values of skin pixels in the RGB color space for two different viewpoints

Fig. 7 visualizes the color histograms of skin pixels in the training images for three cases: (a) the proposed plane, (b) HS plane and (c) IQ plane. We can see that, in the color histogram based on the proposed plane, skin colors form a much tighter cluster than in HS or IQ planes. The distribution of skin colors is very wide and variant in the HS and IQ planes.

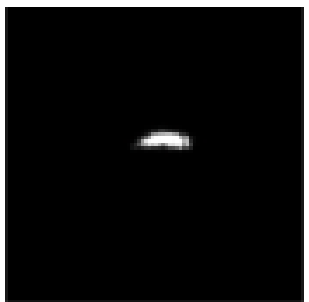

(a) PCA

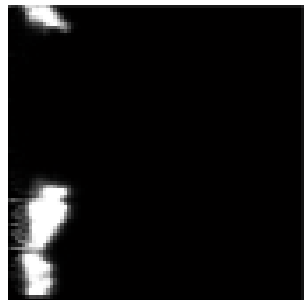

(b) HS plane

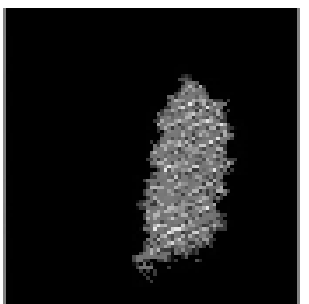

(c) IQ plane

Fig. 7. The visualization of 2D color histograms for three cases: (a) using the proposed plane, (b) HS plane and (c) IQ plane. More frequent values are displayed brighter. 
Fig. 8 shows a set of results for skin color segmentation in the cases of the three planes. As can be seen in Fig. 8 (b) and (c), when HS and IQ planes are used for color segmentation, many non-skin pixels are classified as skin pixels under illumination changes. This indicates that they were found in the entries of the color histogram computed offline. The main reason for the errors is because the initial distribution of HS and IQ values of skin pixels is not valid any longer as the illumination changes. In contrast, the segmentation result using the proposed plane experimentally shows that the color histogram based on the proposed plane does not vary as much as HS and IQ planes. Based on the experimental results, we claim that the proposed plane has much less problem of color constancy. These results indicate that color histograms based on the HS or IQ planes need to be updated from time to time while color histogram based on the proposed plane does not.

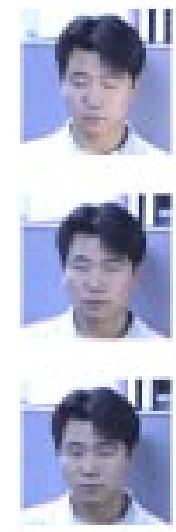

(a)
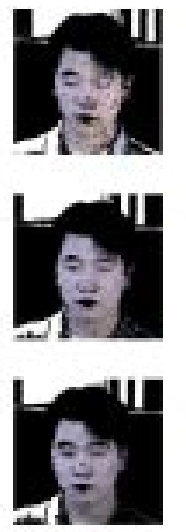

(b)
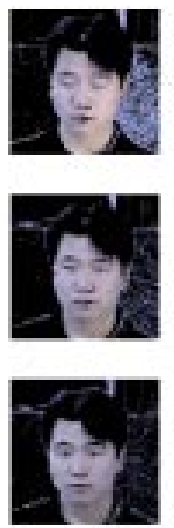

(c)
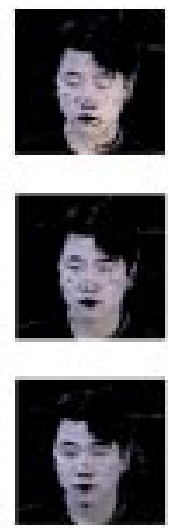

(d)

Fig. 8. Segmentation results: (a) test images (b) for the HS plane (c) for the IQ plane , and (d) for the proposed plane

\section{Conclusions}

Based on the fact that skin colors in the RGB color space are approximately distributed in a linear fashion, we have proposed a novel but simple 2D plane in the RGB color space for skin color segmentation that employs color histogram lookup. The RGB color values of the input color image do not need to be converted into HSI or YIQ color coordinates that have popularly been used for color segmentation. The proposed 2D color plane is formed by the two axes of an RGB color space that correspond to two directions with smallest spread of RGB values of skin pixels. The experimental results show that the performance of skin color segmentation using the proposed plane is much more robust to illumination changes compared to those using HS or IQ planes.

Acknowledgements. This work was supported by grant number R01-1999-00339 from the Basic Research program of the Korean Science and Engineering Foundation. 


\section{References}

1. R. Kjeldsen and J. Kender: Finding Skin in Color Images. International Conference on Automatic Face and Gesture Recognition, 1995.

2. F. Quek, T. Mysliwiec, and M. Zhao, FingerMouse: A Freehand Pointing Interface. Proceedings of IEEE International Conference on Automatic Face and Gesture Recognition, pp. 372-376, 1995.

3. Leonid Sigal, Stan Sclaroff, and Vassilis Athitsos: Estimation and Prediction of Evolving Color Distributions for Skin Segmentation Under Varying Illumination. Proceedings of IEEE Conference on omputer Society Conference on Computer Vision and Pattern Recognition, pp. 152-159, 2000.

4. Rogerio Schmidt Feris, Teofilo Emidio de Campos, and Roberto Marcondes Cesar Junior: Detection and Tracking of Facial Features in Video Sequences. Springer-Verlag press MICAI-2000, pp. 197-206, 1793.

5. Richard P. Schumeyer and Kenneth E. Barner: A Color-Based Classifier for Region Identification in Video. Proceedings of the Visual Communications and Image Processing to (VCIP 98) Conference, vol. 3309, pp. 189-200.

6. Gang Xu and Takeo Sugimoto: Rits Eye: A Software-Based System for Realtime Face Detection and Tracking Using Pan-Tilt-Zoom Controllable Camera. Proceedings of 14th International Conference on Pattern Recognition, pp. 1194-1197, 1998.

7. Gary R. Bradski: Computer Vision Face Tracking For Use in a Perceptual User Interface. Intel Technology Journal Q2, 1998.

8. M. J. Swain and D. H. Ballard: Color Indexing. International Journal of Computer Vision, pp. 11-32, 1991.

9. Michael J. Jones and James M. Rehg, "Statistical Color Models with Application to Skin Detection", Proceedings of IEEE Conference on Computer Vision and Pattern Recognition, pp. 274-280, 1999.

10. David Saxe and Richard Foulds: Toward Robust Skin Identification in Video Images. Proceedings of the 2nd International Conference on Automatic Face and Gesture Recognition, pp. 379-384, 1996.

11. Jung Soh, Ho-Sub Yoon, Min Wang and Byung-Woo Min: Locating Hands in Complex Images Using Color Analysis. IEEE International Conference on Computational Cybernetics and Simulation, pp. 2142-2146, 1997.

12. M. Mottleb and A. Elgammal: Face Detection in Complex Environment from Color Images, International Conference on Pattern Recognition, pp 622-626, 1999. 